\title{
Stresses in Curved Beams Made of Heterogeneous Materials
}

\section{László Kiss and György Szeidl}

Department of Mechanics, University of Miskolc, H-3515 Miskolc-Egyetemváros, Hungary

\section{Abstract \\ The main objective of the present paper is a generalization of some classical results for curved beams made of heterogeneous materials. We consider a beam made of nonhomogeneous, isotropic, linearly elastic material. The elastic parameters depend on the cross-sectional coordinates only. Our investigations include the determination of the normal stress (i.e., the generalization of the Grashof formula), the shearing stress and the curvature change. Interestingly, our newly established formulae have the same structure as the classical ones. We conclude with numerical examples which illustrate the applicability of our results.}

Publication History:

Received: March 30, 2015

Accepted: October 31, 2015

Published: November 05, 2015

\section{Keywords:}

Heterogeneous, Curved beam, Pure bending, Grashof formula

\section{Introduction}

The majority of the textbooks on strength of materials deal with curved beams made of homogeneous material. Formulae are presented for the calculation of the normal stress, the shearing stress, the change of curvature and the strain energy stored in the curved beam. In this paper our main objective is to generalize these classical results for a curved beam made of heterogeneous isotropic materials By assumption the elastic parameters, i.e., the Young modulus and the Poisson number, depend on the cross sectional coordinates, but are independent of the axial coordinate. Figure 1 shows a part of the beam and the applied orthogonal curvilinear coordinates. Our most important assumptions are as follows:

1. The displacements and deformations are small;

2. The curved beam has a uniform cross section and a constant radius;

3. The cross section is symmetric with respect to the axis $\zeta$;

4. The Young modulus $E$ and the Poisson number $v$ satisfy the following relations:

$$
E(\eta, \zeta)=E(-\eta, \zeta) ; \quad v(\eta, \zeta)=v(-\eta, \zeta) ;
$$

1. The Young modulus is the same in tension and compression;

2. The magnitude of the normal stress $\sigma_{\xi}$ is much greater than those of the stress components and $\sigma_{\dot{\zeta}} ;$

3. the temperature is constant (there is no heat effect).

These assumptions are associated with the displacement hypotheses detailed in the following section.

\section{Displacement Hypotheses}

The orthogonal curvilinear coordinates are shown in Figure 1. We shall assume that (a) the crosssections are symmetric with respect to the axis [consequently the beam is symmetric with respect to the coordinate plane $(\xi=s, \zeta)]$ (b) the E-weighted first moment of the cross section with respect to the axis - this quantity is denoted by $Q_{e n}$ - is equal to zero:

$$
Q_{\varepsilon \eta}=\int_{A} E(\eta, \zeta) \zeta \mid d A=0
$$

The axis $\xi=s$ intersects the plane of the cross section in the point $\mathrm{Ce}$, which is referred to as the E-weighted center of the cross section (in contrast to the point $\mathrm{C}$, which is the geometrical center of the cross section).

The coordinate line $\xi=s$ is the E-weighted centerline (or centerline in short) of the curved beam, and $\mathrm{s}$ is the corresponding arc coordinate.

We next introduce the concepts of E-weighted area and moment of inertia with respect to the axis

$$
A_{e}=\int_{A} E(\eta, \zeta) d A, \quad I_{e \eta}=\int_{A} E(\eta, \zeta) \zeta^{2} d A .
$$

These concepts have been introduced for a straight beam in paper [1] by Baksa and Ecsedi.

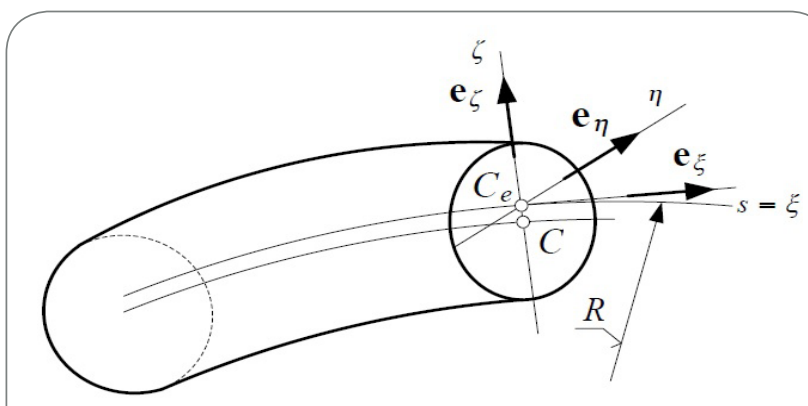

Figure 1: The unit tangent vectors $\mathrm{e}_{\xi}(\mathrm{s}), \mathrm{e}_{n}$ and $\mathrm{e}_{\zeta}(\mathrm{s})$ of the coordinate lines $\xi, n$, and $\zeta$.

The unit tangent vectors $\mathrm{e}_{\xi}(\mathrm{s}), \mathrm{e}_{n}$ and $\mathrm{e}_{\zeta}(\mathrm{s})$ of the coordinate lines $\xi$, $n$, and $\zeta$ are also shown in Figure 1 . Let $R$ be the radius of the E-weighted centerline. It is easy to check that $e_{\xi}(s)$, and $e_{\zeta}(s)$ satisfy the following relations

$$
\frac{d e_{\xi}}{d s}=-\frac{1}{R} e_{\zeta}, \quad \frac{d e_{\zeta}}{d s}=\frac{1}{R} e_{\xi} \quad \text { and } \quad e_{\zeta} \times e_{\xi}=e_{\eta}=\text { constant }
$$

It is also obvious that the operator $\Delta$ takes the form:

$$
\nabla=\frac{\partial}{\partial_{s}} \frac{1}{1+\frac{\zeta}{R}} e_{\xi}+\frac{\partial}{\partial_{\eta}} e \eta+\frac{\partial}{\partial_{\eta}} e_{\eta}+\frac{\partial}{\partial \zeta} e_{\zeta} .
$$

We shall assume that (a) the cross section has a translation and a rigid body rotation about the axis , i.e., it remains a plane surface during the deformation, and (b) the deformed centerline remains perpendicular to the cross section. Under these conditions

"Corresponding Author: Dr. György Szeidl, University of Miskolc, Institute of Applied Mechanics, H-3515 Miskolc-Egyetemváros, Hungary, E-mail: gyorgy.szeidl@uni-miskolc.hu

Citation: Kiss L, Szeidl G (2015) Stresses in Curved Beams Made of Heterogeneous Materials. Int J Mech Syst Eng 1: 107. doi: http://dx.doi. org/10.15344/2455-7412/2015/107

Copyright: @ 2015 kiss et al. This is an open-access article distributed under the terms of the Creative Commons Attribution License, which permits unrestricted use, distribution, and reproduction in any medium, provided the original author and source are credited. 
Citation: Kiss L, Szeidl G (2015) Stresses in Curved Beams Made of Heterogeneous Materials. Int J Mech Syst Eng 1: 107. doi: http://dx.doi.org/10.15344/2455$7412 / 2015 / 107$

$$
u=u_{o}+\psi_{o \eta} \zeta e_{\xi}=\omega_{o} e_{\zeta}+\left(\mu_{o}+\psi_{o \eta} \zeta\right) e_{\xi}
$$

is the displacement field on the cross section, in which $\mathbf{u}_{\mathrm{o}}=u_{\mathrm{o}} \mathbf{e}_{\xi}+$ $w_{\mathrm{o}} \mathbf{e}_{\zeta}$ and $\psi=\psi_{o n} \mathrm{e}_{n}$ are the displacement vector and the rotation on the E-weighted centerline, respectively. As is well known

$$
\psi=-\frac{1}{2}(u \times \nabla)
$$

is the rigid body body rotation. Consequently

$$
\begin{aligned}
\left.\psi\right|_{\zeta=0}=\psi_{o \eta} e_{\eta} & =-\frac{1}{2}\left(\omega_{o} e_{\zeta}+\left(\mu_{o}+\psi_{o \eta} \zeta\right) e_{\xi}\right) \times\left.\left(\frac{\partial}{\partial_{s}} \frac{1}{1+\frac{\zeta}{R}} e_{\xi}+\frac{\partial}{\partial_{\eta}} e_{\eta}+\frac{\partial}{\partial_{\zeta}} e_{\zeta}\right)\right|_{\zeta=0}=(6) \\
& =\frac{1}{2}\left[\frac{1}{1+\frac{\zeta}{R}}\left(\frac{\mu_{o}}{R}-\frac{d \omega_{o}}{d s}\right) e_{\eta}+\psi_{o \eta} e_{\eta}\right]
\end{aligned}
$$

from where

$$
\begin{aligned}
& \psi_{o \eta}=\frac{\mu_{o}}{R}-\frac{d \omega_{o}}{d s} \text { and } \frac{d \psi_{o \eta}}{d s}=\kappa_{o}=-\frac{d}{d_{s}}\left(\frac{d \omega_{o}}{d s}-\frac{\mu_{o}}{R}\right) \\
& u=\omega_{o} e_{\zeta}+\left[\mu_{o}+\left(\frac{\mu_{o}}{R}-\frac{d \omega_{o}}{d s}\right) \zeta\right] e_{\xi} .
\end{aligned}
$$$$
\text { and }
$$

$$
\begin{aligned}
& \text { Since } \\
& u_{o} \nabla=\left[\omega_{o} e_{\zeta}+\left(\mu_{o}+\psi_{o \eta} \zeta\right) e_{\xi}\right] o\left(\frac{\partial}{\partial_{s}} \frac{1}{1+\frac{\zeta}{R}} e_{\xi}+\frac{\partial}{\partial \zeta} e_{\zeta}\right)= \\
& =\frac{1}{1+\frac{\zeta}{R}}\left(\frac{d \mu_{o}}{d s}+\frac{\omega_{o}}{R}+\frac{d \psi_{o \eta}}{d s}\right) e_{\xi} o e_{\xi}((\ldots))
\end{aligned}
$$

where at least one factor in the dyadic products denoted, for brevity, by $((\ldots))$ is perpendicular to $\mathbf{e}_{\xi}$, we have

$$
\begin{aligned}
& \varepsilon_{\xi}=e_{\xi} \cdot \frac{1}{2}\left(u_{o} \nabla+\nabla_{o} u\right) \cdot e_{\xi}= \\
& =\frac{1}{1+\frac{\zeta}{R}}\left(\frac{d \mu_{o}}{d s}+\frac{\omega_{o}}{R}+\frac{d \psi_{o \eta}}{d s} \zeta\right)=\frac{1}{1+\frac{\zeta}{R}}\left(\varepsilon_{o \xi}+\zeta \kappa_{o}\right) . \\
& \text { Here } \\
& \varepsilon_{o \xi}=\frac{d \mu_{o}}{d s}+\frac{\omega_{o}}{R}, \quad \kappa_{o}=\frac{d \psi_{o \eta}}{d s}
\end{aligned}
$$

are the axial strain and the curvature on the E-weighted centerline.

\section{Formulae for the normal stress}

Generalization of the Grashof formula. The E-weighted reduced area, first moment and moment of inertia are defined by the following relations:

$$
\begin{aligned}
& A_{e R}=\int_{A} \frac{R}{R+\zeta} E(\eta, \zeta) d A, \quad Q_{e R}=\int_{A} \frac{R}{R+\zeta} E(\eta, \zeta) \zeta d A \\
& I_{e R}=\int_{A} \frac{R}{R+\zeta} E(\eta, \zeta) \zeta^{2} d A .
\end{aligned}
$$

$$
N=\int \sigma_{\xi} d A, \quad M=\int \zeta \sigma_{\xi} d A
$$

Here, due to the inequality $\sigma_{\xi}>>\sigma_{\eta}, \sigma_{\zeta}, \sigma_{\xi}=E(\eta, \zeta) \varepsilon_{\xi}$, equation is the Hooke law. Upon substitution of the Hook law and then equation (10) into (12) we have

$N=\varepsilon_{o \xi} \int_{A} \frac{R}{R+\zeta} E(\eta, \zeta) d A+\kappa_{o} \int_{A} \frac{R}{R+\zeta} E(\eta, \zeta) \zeta d A=\varepsilon_{o \xi} A_{e R}+\kappa_{o} Q_{e R}(13 \mathrm{a})$

As for the bending moment, in a similar way we obtain

$M=\varepsilon_{o \xi} \int_{A} \frac{R}{R+\zeta} E^{2}(\eta, \zeta) \zeta d A+\kappa_{o} \int_{A} \frac{R}{R+\zeta} E(\eta, \zeta) \zeta^{2} d A=\varepsilon_{o \xi} Q_{e R}+\kappa_{o} I_{e R}$

After solving equation system (13) we have $\epsilon_{0 \xi}$ and $\kappa_{0}$ in terms of the axial force and the bending moment:

$\varepsilon_{o \xi}=\frac{1}{Q_{e R}^{2}-A_{e R} I_{e R}}\left(M Q_{e R}-N I_{e R}\right), \quad \kappa_{o}=\frac{1}{Q_{e R}^{2}-A_{e R} I_{e R}}\left(N Q_{e R}-M A_{e R}\right)(14)$

Let us now substitute these solutions into equation (9) so that we can get the axial strain:

$\varepsilon_{\xi}=\frac{R}{R+\zeta} \frac{1}{A_{e R} I_{e R}-Q_{e R}^{2}}\left[\left(I_{e R}-\zeta Q_{e R}\right) N-M\left(Q_{e R}-\zeta A_{e R}\right)\right]$

With the knowledge of the axial strain

$\sigma_{\xi}=E(\eta, \zeta) \varepsilon_{\xi}=E(\eta, \zeta) \frac{R}{R+\zeta} \frac{1}{A_{e R} I_{e R}-Q_{e R}^{2}}\left[\left(I_{e R}-\zeta Q_{e R}\right) N-M\left(Q_{e R}-\zeta A_{e R}\right)\right](16)$

is the normal stress.

In what follows, we attempt to simplify the above formula for the normal stress. First we shall dlarify how $A_{e R}, Q_{e R}$ and $I_{e R}$ are related to $A_{e}, Q_{e n}$ and $I_{e n}$. Using the power series of fraction $R /(R+\zeta)$ we have

$A_{e R}=\int_{A}\left(1-\frac{\zeta}{R}+\frac{\zeta^{2}}{R^{2}}-\ldots\right) E(\eta, \zeta) d A \cong \underbrace{\int_{A} E(\eta, \zeta) d A}_{A_{e}}-\frac{1}{r} \underbrace{\int_{A}^{A} E(\eta, \zeta) \zeta d A}_{Q_{e \eta}}=$

$=A_{e}-\frac{Q_{e \eta}}{R}=A_{e}$,

$Q_{e R}=\int_{A}\left(1-\frac{\zeta}{R}+\frac{\zeta^{2}}{R^{2}}-\ldots\right) \zeta E(\eta, \zeta) d A \cong$

$$
\cong \underbrace{\int_{A} \zeta E(\eta, \zeta) d A}_{Q_{e \eta}}-\frac{1}{R} \underbrace{\int_{A}^{\zeta^{2} E(\eta, \zeta) d A}}_{I_{e \eta}}=Q_{e \eta}-\frac{I_{e \eta}}{R}=-\frac{I_{e R}}{R}
$$

and

$I_{e R}=\int_{A}\left(1-\frac{\zeta}{R}+\frac{\zeta^{2}}{R^{2}}-\ldots\right) \zeta^{2} E(\eta, \zeta) d A \cong I_{e \eta}$

because $Q_{e n}=0$. For the denominator in equation (16) the following approximation holds

$A_{e R} I_{e R}\left(1-\frac{Q_{e R}{ }^{2}}{I_{e R} A_{e R}}\right) \simeq A_{e R} I_{e R}\left(1-\frac{1}{R^{2}} \frac{I_{e \eta}^{2}}{A_{e R} I_{e R}}\right) \simeq A_{e R} I_{e R}\left(1-\frac{1}{R^{2}} \frac{I_{e \eta}{ }^{2}}{A_{e R}}\right) \simeq A_{e R} I_{e R}$

since

$1>>\frac{1}{R^{2}} \frac{I_{e \eta}}{A_{e R}}$.

Using this result we can rewrite formula (15): 


$$
\begin{aligned}
\varepsilon_{\xi} & \approx \frac{R}{R+\zeta} \frac{1}{A_{e R} I_{e R}}\left[\left(I_{e R}-\zeta Q_{e R}\right) N-M\left(Q_{e R}-\zeta A_{e R}\right)\right]= \\
& =\left(1-\zeta \frac{Q_{e R}}{I_{e R}}\right) \frac{R}{R+\zeta} \frac{N}{A_{e R}}+\left(-\frac{Q_{e R}}{A_{e R} I_{e R}}+\frac{\zeta}{I_{e R}}\right) \frac{R}{R+\zeta} M .
\end{aligned}
$$

If we use approximations (17) we can check the following equations

$\frac{Q_{e R}}{I_{e R}} \simeq-\frac{1}{R} \frac{I_{e \eta}}{I_{e R}} \simeq-\frac{1}{R}, \quad \frac{1}{R} \frac{R}{R+\zeta} \frac{M}{A_{e R}} \simeq \frac{M}{R A_{e R}}$.(21)

Substituting the last two formulae into (20) and the result into the Hooke law we have

$$
\sigma_{\xi}=E(\eta, \zeta)\left(\frac{N}{A_{e R}}+\frac{M}{R A_{e R}}+\frac{M}{I_{e R}} \frac{R}{R+\zeta} \zeta\right) \text {. }
$$

This formula is the generalization of the Grashof formula for the case of cross sectional inhomogeneity.

A formula for the normal stress assuming pure bending. English textbooks contain a formula for the normal stress under the assumption of pure bending - see for instance equation (4.71) p. 224 in [2]. In this subsection it is our aim to generalize the cited equation for heterogenous curved beams. Figure 2 shows the cross section and the geometrical meaning of some notational conventions: $\zeta_{0}$ is the coordinate of the neutral axis with radius $\bar{R}$, and the radius of an arbitrary point $P$ of the cross section $\zeta$ with coordinate is $r=R+\zeta$. Based on equation (16) for pure bending,

$$
\sigma_{\xi}=E(\eta, \zeta) \frac{R}{R+\zeta} \frac{1}{A_{e R} I_{e R}-Q_{e R}^{2}}\left(\zeta A_{e R}-Q_{e R}\right) M
$$

is the formula for the normal stress. We would like to manipulate it into a form similar to the one cited above.

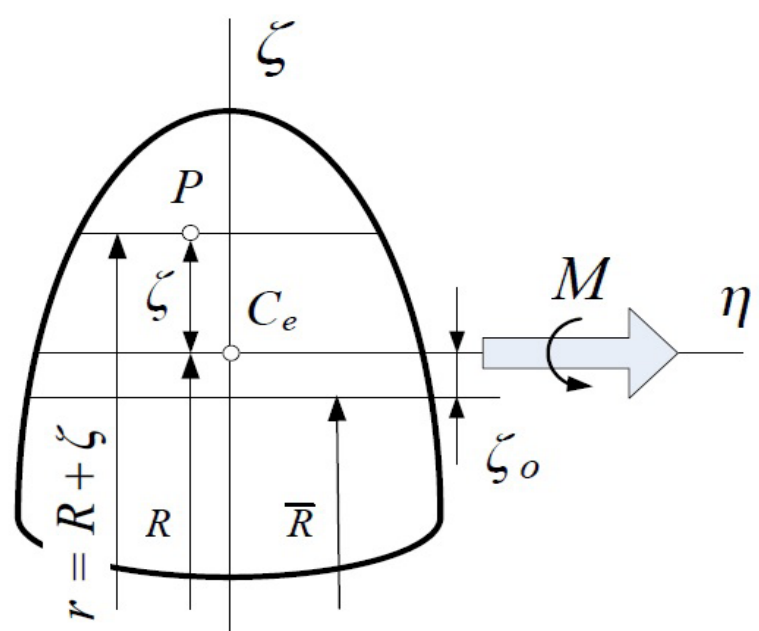

Figure 2: The cross section and the geometrical meaning of some notational conventions.

First we shall determine the location of the neutral axis. It follows from condition $\sigma_{3}=0$ which needs to be satisfied on the neutral axis that

$Q_{e R}=\zeta_{o} A_{e R}$.

Consequently

$Q_{e R}=\left(R+\zeta_{o}-R\right) A_{e R}=(\bar{R}-R) A_{e R}=\bar{R} A_{e R}-R A_{e R}$,

from where

$$
\bar{R}=\frac{Q_{e R}}{A_{e R}}+R
$$

is the radius that belongs to the neutral axis. Upon substitution of $A_{e R}$ and $Q_{e R}$ from (11), the radius $\bar{R}$ of the neutral axis assumes the form

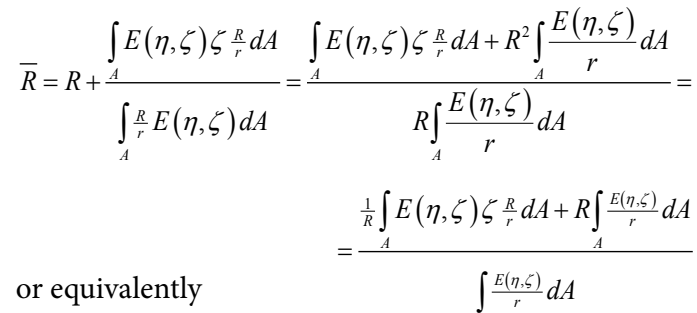

$=\frac{\frac{1}{R} \int_{A} E(\eta, \zeta) \zeta \frac{R}{r} d A+R \int_{A} \frac{E(\eta, \zeta)}{r} d A}{\int_{A} \frac{E(\eta, \zeta)}{r} d A}=\frac{\int_{A} E(\eta, \zeta) d A}{\int_{A} \frac{E(\eta, \zeta)}{r} d A}$

$$
\bar{R}=\frac{\int_{A} E(\eta, \zeta) d A}{\int_{A} \frac{E(\eta, \zeta)}{r} d A}
$$

For $\mathrm{E}(\eta, \zeta)=$ constant, the above equation coincides with formula (4.66) p. 222 in [2]. Now we shall proceed with the determination of the normal stress. Using equation (26), the factor in parentheses in equation (23) can be rewritten into the form

$$
\zeta A_{e R}-Q_{e R}=(r-R) A_{e R}+R A_{e R}-\bar{R} A_{e R}=(r-\bar{R}) A_{e R} .
$$

If we use the inequality $\mathrm{A}_{\mathrm{eR}} \mathrm{I}_{\mathrm{eR}}>\mathrm{S}_{\mathrm{eR}}^{2}$ and substitute back the above difference into equation (23), we obtain

$\sigma_{\xi}=E(\eta, \zeta) \frac{1}{r} M \frac{R}{I_{e R}}(r-\bar{R})$

The last open question is how to transform the fraction $R / I_{e R}$ into a suitable form. The following equation details the transformation step by step:

$$
\begin{aligned}
& I_{e R}=\int_{A} E(\eta, \zeta) \frac{R}{r} \zeta^{2} d A=\int_{A} E(\eta, \zeta) R \frac{R+\zeta-R}{r} \zeta d A= \\
& =\int_{A} E(\eta, \zeta) R \frac{r-R}{r} \zeta d A=R \int_{A} E(\eta, \zeta) \zeta d A-\int_{A} E(\eta, \zeta) R^{2} \frac{1}{r}(r-R) d A= \\
& \underbrace{R Q_{e \eta}}_{0}-\int_{A} E(\eta, \zeta) R^{2} \frac{1}{r}(r-R) d A=-R^{2} \int_{A} E(\eta, \zeta) d A+R^{3} \int_{A} \frac{E(\eta, \zeta)}{r} d A= \\
& =-R^{2} A_{e}+R^{3} \int_{A} \frac{E(\eta, \zeta)}{r} d \overbrace{\overbrace{R-R}^{-\zeta_{0}}}^{=} A_{e}\left(\frac{R^{3}}{\bar{R}}-R^{2}\right)= \\
& =A_{e} R^{2}\left(\frac{R}{\bar{R}}-1\right)=A_{e} R^{2} \overbrace{\left(\frac{R-\bar{R}}{R}\right)}^{A}=-A_{e} \frac{R^{2}}{\bar{R}} \zeta_{o} \text {. }
\end{aligned}
$$

It is worth introducing the notation $e=-\zeta_{0}$. Upon substitution of the result obtained into formula (29) for the normal stress we arrive at its final form:

$$
\sigma_{\xi}=E(\eta, \zeta) \frac{M}{r} \frac{r-\bar{R}}{A_{e} e} .
$$

This equation is the generalization of formula (4.71) p. 224 in [2] valid for the homogenous case for curved beams with cross sectional inhomogeneity.

\section{A formula for the shear stress}


Our goal in this subsection is to derive a closed form solution for calculating the shear stress. We shall use equilibrium equations for this purpose. This approach results in a relatively simple formula; however, it has the disadvantage that the kinematic equations are not satisfied. The basic idea is well known from the theory of straight beams: we divide a short portion of the beam into two parts and then analyze the equilibrium conditions of one part.

Consider Figure 3 which shows a finite portion of the curved beam with cross sectional inhomogeneity. The left cross section with arc coordinate $s_{B}$ is fixed, coordinate $\mathrm{s}>s_{B}$ of the right cross section is regarded as a parameter. We shall use the following assumptions:

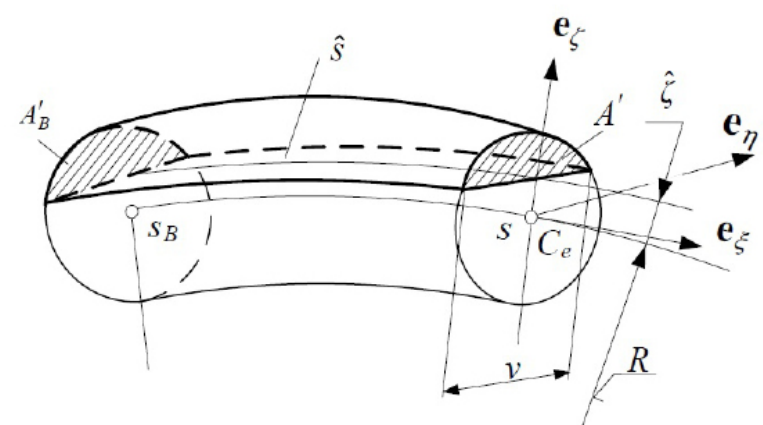

Figure 3: A finite portion of the curved beam with cross sectional inhomogeneity

1. The shear stresses $\tau_{\xi}=\tau_{\eta \xi} e_{\eta}+\tau_{\zeta \xi} \mathrm{e}_{\zeta}$ on the line $\zeta=\hat{\zeta}=$ constant intersect each other in one point which coincides with the intersection point of the tangents to the contour of the cross section at $\zeta=\zeta=$ constant. Consequently, $\tau_{\eta \xi}=-\tau_{\eta \xi}(-\eta)$, which means that $\tau_{\eta \xi}(\eta)$ is an odd function of $\eta$.

2. The shear stress $\tau_{\eta \xi}$ is constant if $\zeta=$ constant.

3. The bending moment $M$ and the shear force $V$ are related to each other via equation

$$
\frac{d M}{d s}=-V
$$

which is an equilibrium condition.

4. The normal stress $\sigma_{\xi}$ can be calculated by equation (22) for which we assume $N=0$ - there is no axial force in the cross section considered.

For calculating the shear stresses $\tau_{\text {k }}$ let us consider the part of the beam with outlines drawn thick in Figure 3. It is bounded by the marked endfaces $A_{B}^{\prime}, A^{\prime}$, the cylinder with radius $\rho_{0}+\hat{\zeta}$ is a part of the lateral surface. By assumption the lateral surface is unloaded.

The equilibrium equation for the part of the beam considered is of the form

$$
\begin{aligned}
\int_{A^{\prime}}\left(\sigma_{\xi} e_{\xi}+\tau_{\xi}\right) d A- & \int_{A_{B}^{\prime}}\left(\sigma_{\xi} e_{\xi}+\tau_{\xi}\right) d A- \\
& -\int_{s_{B}}^{s} \frac{R+\hat{\zeta}}{R} v(\hat{\zeta}) \tau_{\xi \zeta}(\hat{\zeta}) e_{\xi}(\xi) d \xi=0 .
\end{aligned}
$$

If we take into account that the shear stress $-\tau_{\xi \zeta}(\hat{\zeta}) e_{\zeta}(s)$ is constant on

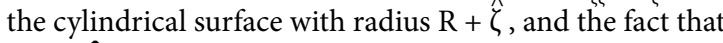

$$
\frac{R+\hat{\zeta}}{R} v(\hat{\zeta}) d \xi=d A
$$

is the surface element, then it follows that the last integral in (33) is the resultant of the shear stresses.

Let us differentiate equation (33) with respect to $s$. Then (a) substitute the derivatives of the unit vectors $\mathbf{e}_{\xi}$ and $\mathbf{e}_{\zeta}$ with respect to $s$ (b) take into account that (1) the integral over $A_{B}^{\prime}$ is constant therefore its derivative is zero; (2) $\tau_{\eta \xi}$ is an odd function of $\eta$ therefore its integral is zero; (3) the derivative of an integral with respect to the upper limit is the integrand. Then

$$
\begin{aligned}
\int_{A} \frac{d \sigma_{\xi}}{d s} e_{\xi} d A-\int_{A} \frac{\sigma_{\xi}}{R} e_{\zeta} d A+\frac{d}{d s} \underbrace{\int_{A} \tau_{\eta \xi} e_{\eta} d A+}_{=0} \\
+\int_{A}\left(\frac{d \tau_{\zeta \xi}}{R} e_{\zeta}+\frac{\tau_{\xi \xi}}{R} e_{\xi}\right) d A-\frac{R+\hat{\zeta}}{R} v(\hat{\zeta}) \tau_{\xi \zeta}(\hat{\zeta}) e_{\zeta}(s)=0 .
\end{aligned}
$$

If we now dot multiply throughout by $\mathrm{e}_{\xi}$ we obtain

$$
\int_{A^{\prime}} \frac{d \sigma_{\xi}}{d s} d A+\int_{A} \frac{\tau_{\zeta \xi}}{R} d A-\frac{R+\hat{\zeta}}{R} v(\hat{\zeta}) \tau_{\xi \zeta}(\hat{\zeta})=0 .
$$

Let $e_{\text {max }}$ be the distance of the top of the cross section from the point $C_{e}$. This is always less than $R$. The area $A^{\prime}$ can be given as a product $v(\hat{\zeta}) h(\hat{\zeta})$ where $h(\hat{\zeta})$ is less than $e_{\text {max }}$. Consequently

$$
\int_{A^{\prime}} \frac{\tau_{\zeta \xi}(\eta, \zeta)}{R} d A \simeq \frac{1}{R} h(\hat{\zeta}) \cup(\hat{\zeta}) \tau_{\xi \zeta}(\hat{\zeta}), \quad \frac{h(\hat{\zeta})}{R} \ll<1
$$

is an upper limit of the second integral in (34). Really if we take into account that the shear stress is taken on the line $\zeta$ (instead of being taken at inner points of $A^{\prime}$ ) we can readily check the validity of the previous statement. On the basis of this estimation the second term in (34) can be neglected if we compare it to the third term. Omitting this term results in the following equation

$$
\int_{A^{\prime}} \frac{d \sigma_{\xi}}{d s} d A=\frac{R+\hat{\zeta}}{R} v(\hat{\zeta}) \tau_{\xi \zeta}(\hat{\zeta})
$$

for the calculation of the shear stress $\tau_{\xi \zeta}(\hat{\zeta})$. After rearranging we obtain the following average value

$\tau_{\xi \zeta}(\hat{\zeta})=\frac{R}{R+\hat{\zeta}} \frac{1}{v(\hat{\zeta})} \int_{A^{\prime}} \frac{d \sigma_{\xi}}{d s} d A$.

Upon substitution of the normal stress from (22) $-N=0$ by assumption - we have

$\tau_{\xi \zeta}(\hat{\zeta})=\frac{R}{R+\hat{\zeta}} \frac{1}{v(\hat{\zeta})} \int_{A} \frac{d}{d s}\left[E(\eta, \zeta)\left(\frac{M}{R A_{e R}}+\frac{M}{I_{e R}} \frac{R}{R+\zeta}\right)\right] d A$

A further transformation yields

$$
\begin{aligned}
& \tau_{\xi \zeta}(\hat{\zeta})=\frac{R}{R+\hat{\zeta}} \frac{1}{v(\hat{\zeta})} \frac{d M}{d s} \int_{A^{\prime}}\left(\frac{E(\eta, \zeta)}{R A_{e R}}+\frac{E}{I_{e R}} \frac{R}{R+\zeta} \zeta\right) d A= \\
& =\frac{R}{R+\hat{\zeta}} \frac{1}{v(\hat{\zeta})} \frac{d M}{d s} \frac{1}{I_{e R}}\left(R \frac{I_{e R}}{R^{2} A_{e R}} \int_{A} E(\eta, \zeta) d A+\int_{A^{\prime}} \frac{R}{R+\zeta} \zeta E(\eta, \zeta) d A\right) .
\end{aligned}
$$

Introducing the notations

$\alpha_{e}=\frac{I_{e R}}{R^{2} A_{e R}} ; \quad Q_{e \eta}{ }^{\prime}=\int_{A^{\prime}} E(\eta, \zeta) \frac{R}{R+\zeta} \zeta d A$

and

$\int_{A^{\prime}} E(\eta, \zeta) d A=A_{e}^{\prime}$

and substituting (32) we obtain

$\tau_{\xi \zeta}(\hat{\zeta})=-\frac{R}{R+\hat{\zeta}} \frac{V}{I_{e R} v(\hat{\zeta})}\left(R \alpha_{e} A_{e}^{\prime}+Q_{e \eta}^{\prime}\right)$

which is a formula for the average value of the shear stress. This result is a generalization of a classical formula valid for curved beams made 
of homogenous material - see pp. 358-359 in [3, 2002].

\section{Curvature change, strain energy}

In the present subsection [the radius of curvature] \{a point on the E-weighted centerline before and after deformation are denoted by $\left[R_{o}\right.$ and $R]\left\{P_{o}\right.$ and $\left.P\right\}$. The angle formed by the tangent to the E-weighted centerline at $P o$ with the horizontal axis is $\psi_{o}$. Its change during deformation is $\psi_{o \eta}$ - the rigid body rotation. The calculation of the curvature change is based on Figure 4 which shows all the quantities mentioned.

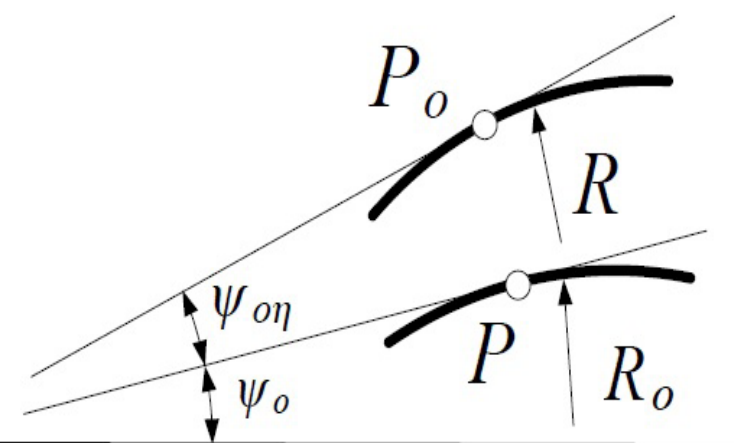

Figure 4: The calculation of the curvature change is based on which shows all the quantities mentioned.

The infinitesimal arc element $\mathrm{d} s$ on the E-weighted centerline before deformation changes to $\mathrm{d} s$. It is clear that

$\varepsilon_{o \xi}=\frac{d s-d s_{o}}{d s_{o}}$

is the axial strain on the E-weighted centerline. Consequently

$d s_{o}=\frac{d s}{1+\varepsilon_{o \xi}}$.

Using the above equation we can manipulate the formula for the curvature change:

$\frac{1}{R}-\frac{1}{R_{0}}=\frac{d\left(\psi_{o}+\psi_{o \eta}\right)}{d s}-\frac{d \psi_{o}}{d s_{o}}=\frac{d\left(\psi_{o}+\psi_{o \eta}\right)}{d s}-\frac{d \psi_{o}}{d s}\left(1+\varepsilon_{o \xi}\right)=\frac{d \psi_{o \eta}}{d s}-\varepsilon_{o \xi \xi} \frac{d \psi_{o}}{d s}$. (42)

Here

$\varepsilon_{o \xi} \frac{d \psi_{o}}{d s}=\varepsilon_{o \xi} \frac{d \psi_{o}}{d s_{o}}\left(1+\varepsilon_{o} \xi\right)=\varepsilon_{o \xi} \frac{d \psi_{o}}{d s_{o}}=\varepsilon_{o \xi} \frac{1}{R_{o}}$.

A comparison of equations (10), (42) and (43) yields

$\frac{1}{R}-\frac{1}{R_{o}}=\kappa_{o}-\varepsilon_{o \xi} \frac{1}{R}$.

Substituting $\kappa_{0}$ from (14) and taking into account that in the present case $N=0$ and $Q_{e R}^{2}<<A_{e R} I_{e R}$ we have

$\frac{1}{R}-\frac{1}{R_{o}}=-\frac{M}{Q_{e R}^{2}-A_{e R} I_{e R}}(A_{e R}+\underbrace{\frac{Q_{e R}}{R}}_{\approx 0}) \simeq \frac{M A_{e R}}{A_{e R} I_{e R}-Q_{e R}^{2}} \simeq \frac{M A_{e R}}{A_{e R} I_{e R}}=\frac{M}{I_{e R}}$

that is

$\frac{1}{R}-\frac{1}{R_{o}}=\frac{M}{I_{e R}}$.

Now we proceed to determine the strain energy stored in the beam. It is not too difficult to check using equation (45) that the angle change $\mathrm{d} \psi$ due to the bending moment is $d \psi=\frac{d s}{R}-\frac{d s_{o}}{R_{o}} \simeq \frac{d s}{R}-\frac{d s}{R_{o}}=\frac{M}{I_{e R}} d s$

Consequently

$d U=\frac{1}{2} M d \psi=\frac{1}{2} \frac{M^{2}}{I_{e R}} d s$

is the work done by the bending moment exerted on an infinitesimal portion of the beam. Let $L$ be the length of the $E$-weighted centerline. After integration

$Q_{e \eta}=Q_{e y}-z_{C} A_{e}=0$.

\section{Numerical examples}

Example 1. Figure 5 shows the cross-section of the curved beam considered. We assume that the beam is subjected to pure bending $\mathbf{M}$ $=M \mathbf{e}_{\eta} ; M=100 \mathrm{Nm}$. The geometric dimensions are all given in Figure 5. The lower part of the beam is made of steel and the upper part of the beam is made of aluminium. The corresponding material parameters are $E_{1}=2: 1 \times 10^{5} \mathrm{MPa}$ and $E_{2}=7 \times 10_{4} \mathrm{MPa}$, in that order. Our aim is to depict graphically the normal stress distribution as a function of $\zeta$ using the three formulae derived in the previous sections. This allows us to compare the various results. It would also be interesting to check the difference between these formulae regarding the radius of the neutral axis.

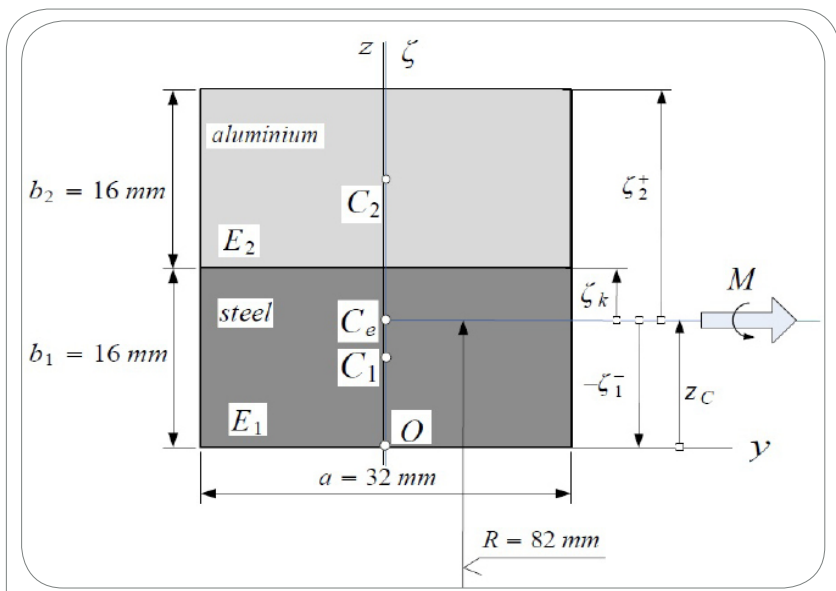

Figure 5: The cross-section of the curved beam considered

First we determine the ordinate $z_{\mathrm{C}}$ of the $E$-weighted centerline in coordinate system $\gamma z$. Since the $E$-weighted first moment of the cross section with respect to the axis $\eta$ should be zero, the following equation holds:

$$
Q_{e \eta}=Q_{e y}-z_{C} A_{e}=0 \text {. }
$$

Consequently

$$
z_{C}=\frac{Q_{e y}}{A_{e}}=\frac{E_{1} \frac{b_{1}}{2} A_{1}+E_{2}\left(b_{1}+\frac{b_{1}}{2}\right) A_{2}}{E_{1} A_{1}+E_{2} A_{2}}=12 \mathrm{~mm} .
$$

With the knowledge of $z_{\mathrm{C}}$ one can read off from Figure 5 that

$$
\zeta_{1}^{-}=-z_{C}=-12 \mathrm{~mm}, \quad \zeta_{k}=4 \mathrm{~mm}, \quad \zeta_{2}{ }^{+}=20 \mathrm{~mm} \text {.(51) }
$$

Before computing the stresses sought we shall set up appropriate formulae for the $E$-weighted geometrical quantities $A_{e R^{\prime}}, Q_{e R^{\prime}}, I_{e \eta}$ and $I_{e R}$. Recalling equation (11)1 we can write

$$
A_{e R}=\int_{L} \frac{R+\zeta-\zeta}{R+\zeta} E(\eta, \zeta) a d \zeta=\underbrace{\int_{A} E(\eta, \zeta) d A-E_{1} a}_{A} \int_{\zeta_{1}}^{\zeta_{1}} \frac{\zeta}{R+\zeta} d \zeta-E_{2} a \int_{\zeta_{1}}^{\zeta_{1}^{+}} \frac{\zeta}{R+\zeta} d \zeta=
$$


Citation: Kiss L, Szeidl G (2015) Stresses in Curved Beams Made of Heterogeneous Materials. Int J Mech Syst Eng 1: 107. doi: http://dx.doi.org/10.15344/2455$7412 / 2015 / 107$

Page 6 of 8

$=A_{e}-E_{1} a\left[\zeta-R \ln \left(1+\frac{\zeta}{R}\right)\right]\left|\begin{array}{l}\zeta_{\kappa} \\ \zeta_{1}^{-}\end{array}-E_{2} a\left[\zeta-R \ln \left(1+\frac{\zeta}{R}\right)\right]\right| \zeta_{\zeta_{\kappa}}^{\zeta_{2}}=$
$=A_{1} E_{1}+A_{2} E_{2}+a\left[\left(E_{2}-E_{1}\right) \zeta_{\kappa}+E_{1} \zeta_{1}^{-}-E_{2} \zeta_{2}^{+}\right]+$
$+a\left[\left(E_{2}-E_{1}\right) R \ln \left(1+\frac{\zeta \kappa}{R}\right)-E_{1} R \ln \left(1-\frac{\zeta_{1}^{-}}{R}\right)+E_{2} R \ln \left(1+\frac{\zeta_{2}^{+}}{R}\right)\right](52)$

Regarding the E-weighted reduced first moment of the crosssection, equation $(11)_{2}$ yields

$$
\begin{aligned}
Q_{e R} & =\int \frac{R+\zeta-\zeta}{R+\zeta} E(\eta, \zeta) \zeta a d \zeta=\underbrace{Q_{e \eta}}_{=0}-\int \frac{\zeta^{2} E(\eta, \zeta)}{R+\zeta} a d \zeta=-\int \frac{\zeta^{2} E(\eta, \zeta)}{R+\zeta} a d \zeta= \\
= & -E_{1} a\left[\frac{1}{2}\left(\zeta_{\kappa}\right)^{2}-\zeta_{K} R+R^{2} \ln \left(R+\zeta_{\kappa}\right)-\frac{1}{2}\left(\zeta_{1}^{-}\right)^{2}+\zeta_{1}^{-} R-R^{2} \ln \left(R+\zeta_{1}^{-}\right)\right]- \\
& -E_{2} a\left[\frac{1}{2}\left(\zeta_{2}^{+}\right)^{2}-\zeta_{2}^{+} R+R^{2} \ln \left(R+\zeta_{2}^{+}\right)-\frac{1}{2}\left(\zeta_{\kappa}\right)^{2}+\zeta_{K} R-R^{2} \ln \left(R+\zeta_{\kappa}\right)\right](53)
\end{aligned}
$$

Using the parallel axis theorem, we can determine $I_{e \eta}$ as

$I_{e \eta}=\int_{A} E(\eta, \zeta) \zeta^{2} d A=E_{1} \int_{A_{1}} \zeta^{2} d A+E_{2} \int_{A_{2}} \zeta^{2} d A=$

$=E_{1}\left[\frac{a b_{1}^{3}}{12}+\left(z_{C}-\frac{b_{1}}{2}\right)^{2} a b_{1}\right]+E_{2}\left[\frac{a b_{2}^{3}}{12}+\left(b_{1}-z_{C}+\frac{b_{2}}{2}\right)^{2} a b_{2}(\$ 4)\right.$

Therefore, recalling (11) $)_{3}$ and utilizing equation (54) we can establish a formula for the $E$-weighted reduced moment of inertia:

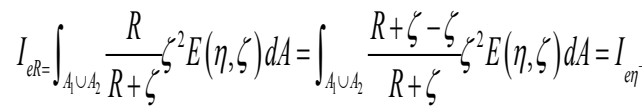

$-\int_{A_{1} \cup A_{2}} \frac{\zeta^{3}}{R+\zeta} E(\eta, \zeta) d A=I_{\text {en }}+a\left(E_{2}-E_{1}\right)\left(\zeta_{K} R^{2}-\frac{1}{2}\left(\zeta_{K}\right)^{2} R-R^{3} \ln \left(R+\zeta_{K}\right)+\frac{1}{3}\left(\zeta_{K}\right)^{3}\right)-$

$-E_{1} a\left(-\frac{1}{3}\left(\zeta_{1}^{-}\right)^{3}+\frac{1}{2}\left(\zeta_{1}^{-}\right)^{2} R-\zeta_{1}^{-} R^{2}+R^{3} \ln \left(R+\zeta_{1}^{-}\right)\right)-$

$-a E_{2}\left(+\frac{1}{3}\left(\zeta_{2}^{+}\right)^{3}-\frac{1}{2}\left(\zeta_{2}^{+}\right)^{2} R+\zeta_{2}^{+} R^{2}-R^{3} \ln \left(R+\zeta_{2}^{+}\right)\right)$.

Substituting now $\mathrm{a}, \mathrm{b}_{1}, \mathrm{~b}_{2}, \mathrm{R}, \mathrm{A}_{1}, \mathrm{E}_{1}, \mathrm{~A}_{2}, \mathrm{E}_{2}, \zeta_{\mathrm{k}}, \zeta_{1}$ and $\zeta_{2}{ }_{2}$ into equations (52)-(55) we obtain the following numerical values:

$A_{e}=1.4336 \times 10^{8} \mathrm{~N}, A_{e R}=1.4477 \times 10^{8} \mathrm{~N}, Q_{e R}=-1.1588 \times 10^{8} \mathrm{Nmm}$, $I_{e \eta}=9.9396 \times 10^{9} \mathrm{Nmm}^{2}, I_{e R}=9.5024 \times 10^{9} \mathrm{Nmm}^{2}$.

With these results we can compute the normal stress 6 using Eqs. (16) - this formula has no simplifications, it is exact under the displacement and stress hypotheses -, (22) - this is a generalization of the Grashof formula - and (31) - this a generalization of the formula that can be found in English textbooks on Strengths of Materials. The computational results are presented in Table 1 and graphically in Figure 6. It is clear that the results obtained by the use of the Grashof formula and by Eq. (31) almost coincide.

As regards Figure 6 the graphs representing the exact solution, the solution obtained by the use of Eq. (22) and the solution calculated with Eq. (31) are drawn in blue, red and green. Observe that the red and green curves coincide almost completely.

\begin{tabular}{|l|l|l|l|}
\hline & \multicolumn{3}{|l|}{ Computed by using Normal stress } \\
\hline$\zeta[\mathrm{mm}]$ & Eq. $(16)$ & Eq. $(22)$ & Eq. $(31)$ \\
\hline-12.0 & -28.693 & -29.332 & -29.281 \\
\hline-11.0 & -25.763 & -26.342 & -26.291 \\
\hline-10.0 & -22.915 & -23.435 & -23.384 \\
\hline-9.0 & -20.144 & -20.608 & -20.557 \\
\hline-8.0 & -17.448 & -17.857 & -17.806 \\
\hline-7.0 & -14.824 & -15.179 & -15.128 \\
\hline-6.0 & -12.270 & -12.573 & -12.521 \\
\hline-5.0 & -9.781 & -10.033 & -9.982 \\
\hline-4.0 & -7.357 & -7.559 & -7.507 \\
\hline-3.0 & -4.993 & -5.148 & -5.096 \\
\hline-2.0 & -2.689 & -2.796 & -2.744 \\
\hline-1.0 & -0.442 & -0.503 & -0.451 \\
\hline 0.0 & 1.751 & 1.734 & 1.786 \\
\hline 1.0 & 3.890 & 3.917 & 3.970 \\
\hline 2.0 & 5.979 & 6.048 & 6.101 \\
\hline 3.0 & 8.018 & 8.129 & 8.183 \\
\hline 4.0 & 10.011 & 10.163 & 10.216 \\
\hline 4.0 & 3.337 & 3.387 & 3.405 \\
\hline 5.0 & 3.986 & 4.049 & 4.067 \\
\hline 6.0 & 4.620 & 4.696 & 4.714 \\
\hline 7.0 & 5.239 & 5.329 & 5.347 \\
\hline 8.0 & 5.845 & 5.947 & 5.965 \\
\hline 9.0 & 6.438 & 6.552 & 6.570 \\
\hline 10.0 & 7.018 & 7.144 & 7.162 \\
\hline 11.0 & 7.585 & 7.227 & 7.741 \\
\hline 12.0 & 8.141 & 8.289 & 8.307 \\
\hline 13.0 & 8.684 & 8.844 & 8.862 \\
\hline 14.0 & 9.216 & 9.387 & 9.405 \\
\hline 15.0 & 9.738 & 9.919 & 9.937 \\
\hline 16.0 & 10.248 & 10.440 & 10.458 \\
\hline 17.0 & 10.749 & 10.951 & 10.969 \\
\hline 18.0 & 11.239 & 11.451 & 11.469 \\
\hline 19.0 & 11.719 & 11.941 & 11.960 \\
\hline 20.0 & 12.191 & 12.422 & 12.440 \\
\hline & $f 919$ & & \\
\hline & & & \\
\hline
\end{tabular}

Table 1: Grashof formula computational results.

As for the ordinate of the neutral axis, by setting $\sigma_{\xi}(\zeta)=0$,Eqs. (16), (22) and (31) yield $-0.8004 \mathrm{~mm},-0.7771 \mathrm{~mm}$ and $-0.7845 \mathrm{~mm}$, respectively. We note that the latter result is exactly the same as the value that can be obtained by using Eq. (26).

Example 2. Figure 7 shows a cross-section of the curved beam considered - observe that the beam is the same as in the previous example. Let us assume that the cross-section is subjected to a shear force $V=10 \mathrm{kN}$. The shear stress can be calculated using Eqs. (38) and (39). Upon substitution of and (56) into (38a) we have

$$
\alpha=\frac{I_{e R}}{R^{2} A_{e R}}=\frac{9.5024 \times 10^{9}}{82^{2} \times 1.447731712 \times 10^{8}}=9.7615 \times 10^{-3} .
$$




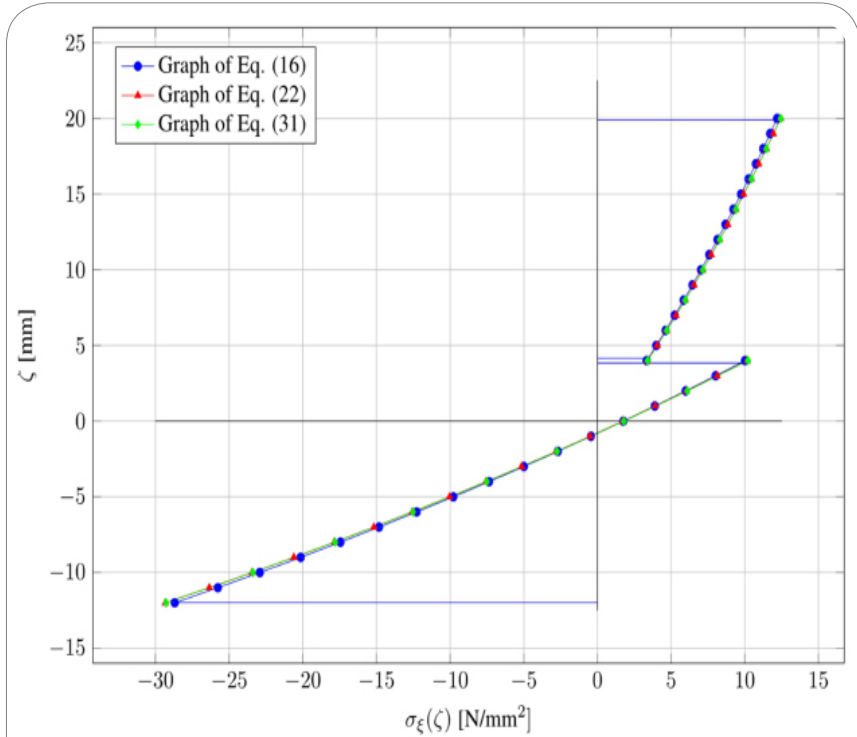

Figure 6: Graphical representation of Grashof formula computational results.

If $\hat{\zeta} \in[4,20]$ then

$A_{e}^{\prime}=\int_{A^{\prime}} E_{2} d A=70000 \times 32 \times \int_{\hat{\zeta}}^{20} d \zeta=44800000-2240000 \hat{\zeta} N$.

We get in the same way that

$Q_{e \eta}{ }^{\prime}=\int_{A} E_{2} \frac{R}{R+\zeta} \zeta d A=70000 \times 32 \times \int_{\hat{\zeta}}^{20} \frac{82}{82+\zeta} d \zeta=$

$=2.24 \times 10^{6} \times(1640-6724 \ln 2-6724 \ln 3-6724 \ln 17-82 \hat{\zeta}+6724 \ln (82+\hat{\zeta}))=$

$=2.24 \times 10^{6} \times(-29458.3172-82 \hat{\zeta}+6724 \ln (82+\hat{\zeta})) \mathrm{Nmm}$.

With $\alpha_{e}, A_{e}(\hat{\zeta})$ and $Q_{e \eta}^{\prime}(\hat{\zeta})$ we can determine the shear stress from

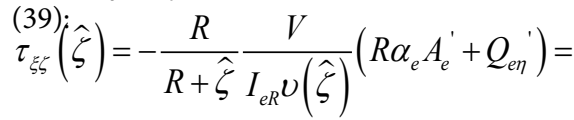

$=-\frac{82}{82+\hat{\zeta}} \times \frac{50000}{9.5024 \times 10^{9} \times 32}\left[82 \times 9.7615 \times 10^{-3} \times(44800000-2240000\right.$
$=\frac{3.7150}{82+\hat{\zeta}} \times\left(-2.0195 \times 10^{3} \hat{\zeta}-7.2099 \times 10^{5}+1.64 \times 10^{5} \ln (82+\hat{\zeta})\right) \mathrm{N} / \mathrm{mm}^{2}$

if $\zeta \in[-12,4]$ then

$A_{e}^{\prime}=\int_{A} E_{1} d A=210000 \times 32 \times \int_{-12}^{\hat{\zeta}} d \zeta=6720000 \hat{\zeta}+80640000 N$,

$Q_{e \eta}{ }^{\prime}=\int_{A} E_{1} \frac{R}{R+\zeta} \zeta d A=210000 \times 32 \int_{-12}^{\hat{\zeta}} \frac{82 \zeta}{82+\zeta} d \zeta=$

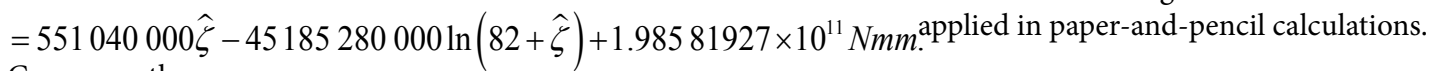

Consequently

$$
\begin{aligned}
& \tau_{\xi \zeta}(\hat{\zeta})=-\frac{R}{R+\widehat{\zeta}} \frac{V}{I_{e R} v(\hat{\zeta})}\left(R \alpha_{e} A_{e}^{\prime}+Q_{e \eta}{ }^{\prime}\right)= \\
& =-\frac{82}{82+\widehat{\zeta}} \times \frac{50000}{9.5024 \times 10^{9} \times 32}\left[82 \times 9.7615 \times 10^{-3} \times(6720000-80640000)+\right. \\
& =\frac{3.7150}{82+\widehat{\zeta}} \times\left(-2.0195 \times 10^{3} \hat{\zeta}-7.2099 \times 10^{5}+1.64 \times 10^{5} \ln (82+\hat{\zeta})\right) \mathrm{N} / \mathrm{mm}^{2}
\end{aligned}
$$$$
=-\frac{82}{82+\widehat{\zeta}} \times \frac{50000}{9.5024 \times 10^{9} \times 32}\left[82 \times 9.7615 \times 10^{-3} \times(6720000-80640000)+551040000 \widehat{\zeta}-45185280000 \ln (82+\widehat{\zeta})+1.98581927 \times 10^{11}\right]=
$$

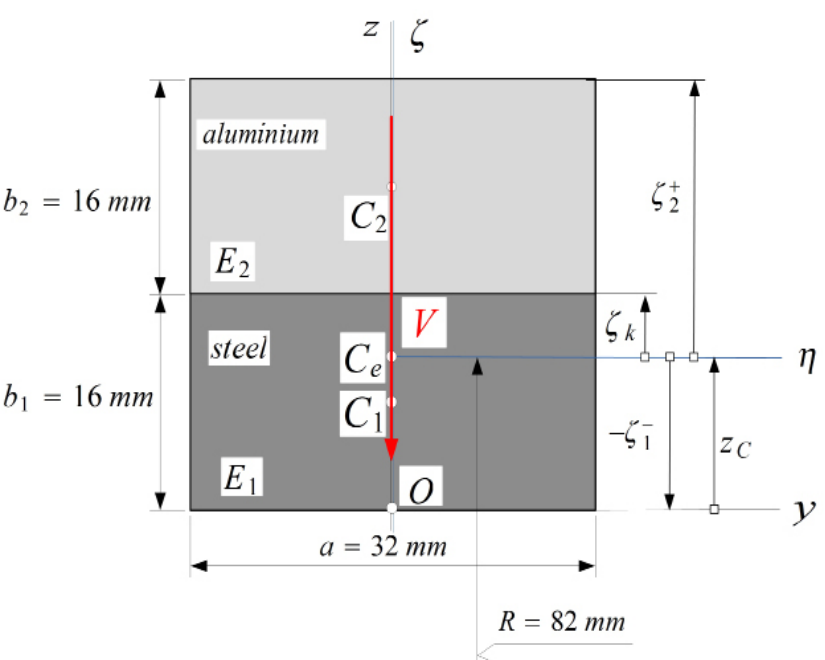

Figure 7: Cross-section of the curved beam.

The values in Table 2 are computed using equations (57) and (58).

We should remark that formula (39) for the shear stress provides an average value - it has been established by using equilibrium conditions. Consequently, the values obtained using this equation are in all probability more accurate if the modulus of elasticity is independent of $\eta$, i.e., if it depends on $\zeta$ only.

\section{Conclusion}

In this paper we have generalized some classical results valid for curved beams made of homogeneous and isotropic material for the case when the curved beam is made of heterogeneous and isotropic material, under the assumption that the elastic parameters, i.e., the Young modulus and the Poisson number, depend on the crosssectional coordinates, but are independent of the axial coordinate. We remark that the Poisson number has played no role in the investigations.

By applying the well known displacement hypotheses we have established (1) three formulae for calculating the normal stress, (2) a formula for the shear stress, and (3) formulae for the change of curvature and the strain energy stored in the beam.

Except the very first formula for the normal stress -- see Eq. (16) -- all the others are generalizations of classical results and each can be

Two numerical examples are presented to illustrate the applicability of the formulae. 
Citation: Kiss L, Szeidl G (2015) Stresses in Curved Beams Made of Heterogeneous Materials. Int J Mech Syst Eng 1: 107. doi: http://dx.doi.org/10.15344/2455$7412 / 2015 / 107$

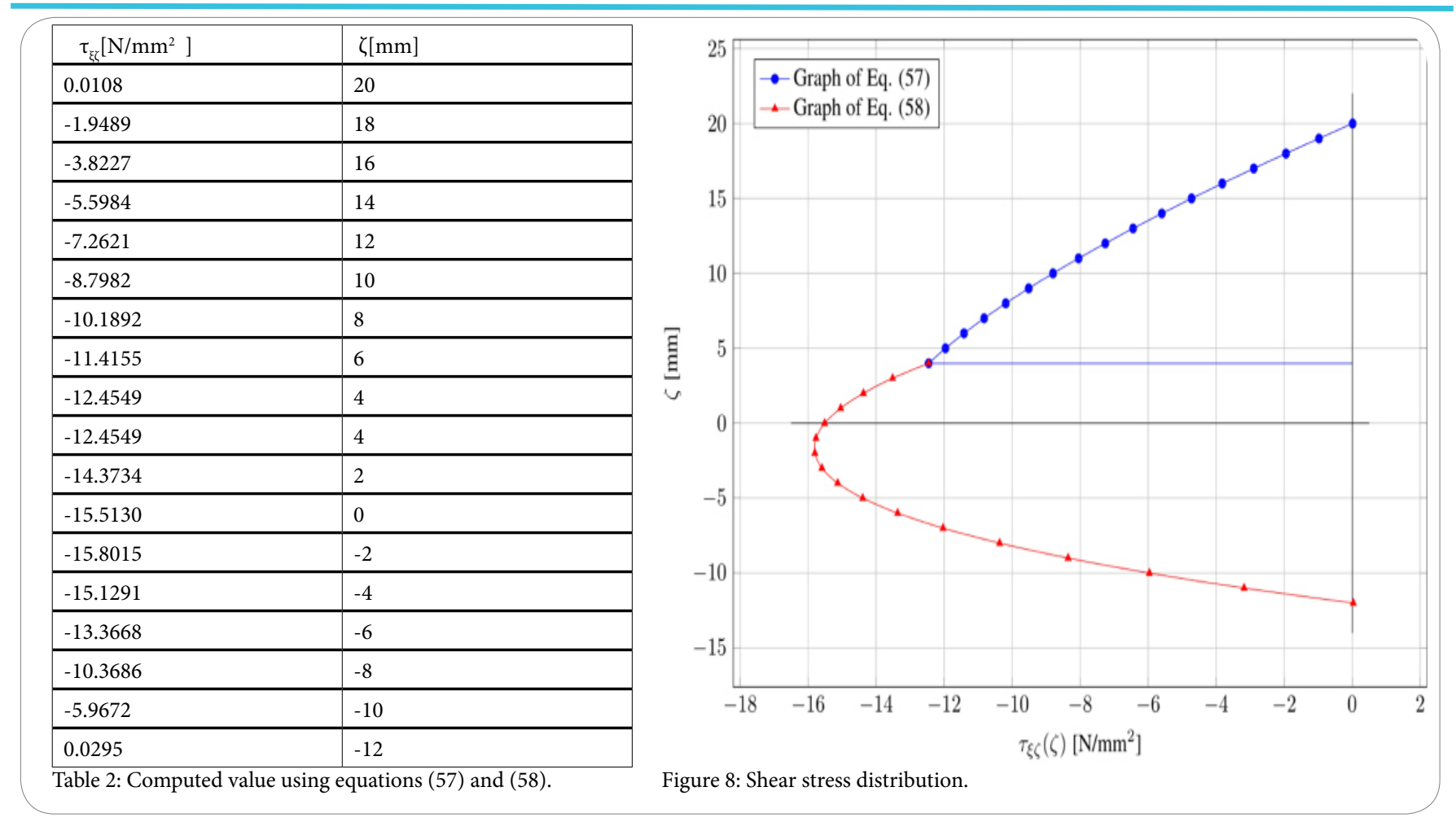

\section{Competing Interests}

The authors declare that they have no competing interests.

\section{Author Contributions}

Both the authors substantially contributed to the study conception and design as well as the acquisition and interpretation of the data and drafting the manuscript.

\section{References}

1. Kozák I, Szeidl Gy (2012) Chapters from the Strength of Materials, Miskolci Egyetem.

2. Beer FP, Johnston ER (1987) Mechanics of Materials, Mc Graw Hill, Metric edition.

3. Csizmadia B, Nándori E (2002) Engineering Mechanics: Strength of Materials. Nemzeti Tankönyvkiadó.

4. Boresi AP, Smith RJ, Sidebottom OM (1993) Advanced mechanics of materials. John Wiley \& Sons, Inc.

5. Aimin $Y(2003)$ Solutions of the integral equations for shearing stresses in two-material curved beams. Mechanics Research Communications 31:137-146.

6. Ecsedi I, Dluhi K (2005) A linear model for the static and dynamic analysis of non-homogeneous curved beams. Applied Mathematical Modelling 29: 1211-1231.

7. Ecsedi I, Lengyel AJ (2015) Curved composite beam with interlayer slip loaded by radial load. Curved and Layered Structures 2: 50-58.

8. Baksa A, Ecsedi I (2009) A note on the pure bending of nonhomogenous prismatic bars. International Journal of Mechanical Engineering Education 37: 118-129. 